\title{
Die Schwingungsspektren von Natrium- und Zinkhydrazid
}

\author{
Von J. Goubeau und U. Kull ${ }^{1}$ ) \\ Professor H.W. Kohlschütter zum 60. Geburtstage gewidmet
}

\section{Inhaltsübersicht}

Nach bekannten Verfahren wurden Natrium- und Zinkhydrazid dargestellt. Das Zinksalz hat im Gegensatz zu den bisherigen Angaben die Zusammensetzung $\mathrm{Zn}\left(\mathrm{N}_{2} \mathrm{H}_{3}\right)_{2}$. Von beiden Verbindungen wurden die IR-Spektren aufgenommen und die Bindungsverhältnisse diskutiert. Beim Natriumsalz zeigt sich die erwartete Lockerung der N-N-Bindung gegenüber Hydrazin. Im Zinkhydrazid wird aus der Verfestigung der N-N-Bindung gegenüber Hydrazin auf Komplexbildung mit kovalenter Bindung $\mathrm{Zn}-\mathrm{N}$ geschlossen.

\section{Summary}

Sodium and zine hydrazide were prepared by known procedures. For the zine salt, the composition $\mathrm{Zn}\left(\mathrm{N}_{2} \mathrm{H}_{3}\right)_{2}$ was found, contrary to previous claims. IR-spectra of both substances were taken and discussed with regard to bond properties. The sodium salt shows the expected weakening of the $\mathrm{N}-\mathrm{N}$-bond compared to $\mathrm{N}_{2} \mathrm{H}_{4}$. In the zine salt, having stronger $\mathrm{N}-\mathrm{N}$-bonds than $\mathrm{N}_{2} \mathrm{H}_{4}$, covalent $\mathrm{Zn}-\mathrm{N}$-bonds are assumed.

Bereits an einer Reihe von Beispielen wurden Änderungen der Kraftkonstanten beobachtet, wenn sich die Zahl der freien Elektronenpaare an den beiden gebundenen Atomen ändert. Dies läßt sich mit verschiedener Hybridisierung erklären; bei Zunahme der freien Elektronenpaare mit einer Abnahme des s-Anteils bzw. mit einer Zunahme des p-Anteils, das mit einem Absinken des Wertes für das Überlagerungsintegral verbunden ist. Die bisherigen Beispiele der Hydrazinverbindungen sollten durch die Untersuchung von Hydraziden erweitert werden. Von diesen sind bisher nur das Natrium- $\mathrm{NaN}_{2} \mathrm{H}_{3}$ und Zinkhydrazid $\mathrm{ZnN}_{2} \mathrm{H}_{2}$ genauer beschrieben worden $\left.\left.\left.{ }^{2}\right)^{3}\right)^{4}\right)^{5}$ ), weshalb wir uns auf diese beiden Beispiele beschränkten.

1) Uldich KuLt, Staatsexamenarbeit, Technische Hochschule Stuttgart 1961.

2) T. W. B. Welsh, J. Amer. chem. Soc. 37, 497 (1915).

${ }^{3}$ ) W. Schlenk u. Th. Weichselfelder, Ber. dtsch, chem. Ges, 48, 669 (1915).

1) E. Ebler u. R. L. Krause, Ber. dtsch. chem. Ges. 43, 1690 (1910).

$\left.{ }^{5}\right)$ L. F. Aunrieth u. B. Ack Erson Oga, The Chemistry of Hydrazine, New York 1951. 


\section{Die Schwingungsspektren der Hydrazide}

Für die Natrium-Verbindung darf mit weitgehend salzartigem Charakter gerechnet werden, also mit der Gegenwart des freien HydrazidIons $\mathrm{N}_{2} \mathrm{H}_{3}-$ in erster Näherung. Da schon im Hydrazin die windschiefe Form mit der Symmetrie $\mathrm{C}_{3}$ vorliegt, $\mathrm{mu}$ auch für das Ion mit derselben Symmetrie gerechnet werden, d. h. alle neun möglichen Schwingungen, die sich auf die Schwingungsklassen $A^{\prime}(6)$ und $A^{\prime \prime}$ (3) verteilen, sind raman- und infrarot-aktiv. Von diesen neun Schwingungen sind 4 Valenzschwingungen und う Deformationssehwingungen. Die Übersicht über die Schwingungen des $\mathrm{N}_{2} \mathrm{H}_{3}$-Ions ist in Tab. 1 gegeben.

Tabelle 1

Schwingungen des $\mathrm{N}_{2} \mathrm{H}_{3}^{-}$-Ions

\begin{tabular}{c|c|c}
\hline & $\mathrm{A}^{\prime}$ & $\mathrm{A}^{\prime \prime}$ \\
\hline $\begin{array}{c}\text { Valenzschwingungen } \\
\text { Deformationsschwin- } \\
\text { gungen }\end{array}$ & $v_{\mathrm{s}} \mathrm{NH}_{2}, v \mathrm{NH}, \quad v \mathrm{NN}$ & $v_{\text {as }} \mathrm{NH}_{2}$ \\
& $\delta \mathrm{NH}_{2}, \delta \mathrm{NNH}_{2}, \delta \mathrm{NNH}$ & $\varrho \mathrm{NH}_{2}, \tau$
\end{tabular}

Die Analyse des von uns erhaltenen Zinkhydrazids ergab im Gegensatz $\mathrm{zu}$ den Literaturangaben ${ }^{4}$ ) nicht die Zusammensetzung $\mathrm{ZnN}_{2} \mathrm{H}_{2}$, sondern $\mathrm{Zn}\left(\mathrm{N}_{2} \mathrm{H}_{3}\right)_{2}$. Deshalb können dieselben Überlegungen auch für das Zinksalz angewandt werden. Zum Unterschied vom Natriumsalz wurden jedoch im Zinksalz starke Frequenzen im Caesiumbromidbereich beobachtet, die Schwingungen von $\mathrm{Zn}-\mathrm{N}$-Bindungen zuzuordnen sind. Es handelt sich also um kovalente $\mathrm{Zn}-\mathrm{N}$-Bindungen.

In Tab. 2 sind die Ergebnisse für beide Verbindungen den Ergebnissen an Hydrazin gegenïbergestellt und die getroffene Zuordnung gegeben, die sich eng an die des Hydrazins anschließt.

Den Erwartungen entsprechend wurden drei NH-Valenzfrequenzen beobachtet (Tab. 2). Bei deren Zuordnung war die Tatsache maßgebend, daß die Differenz zwischen $v_{\mathrm{ars}}$ und $v_{\mathrm{S}} \mathrm{NH}_{2}$ immer in der Größenordnung von $\sim 50 \mathrm{~cm}^{-1}$ liegt, z. B. $\left.\mathrm{NH}_{2} \mathrm{OH} 49^{6}\right),\left(\mathrm{CH}_{3}\right)_{2} \mathrm{CHNH}_{2}$ $\left.55^{7}\right), \mathrm{CH}_{3} \mathrm{NH}_{2} 57^{6}$ ) und $\left.\mathrm{KNH}_{2} 58 \mathrm{~cm}^{-18}\right)$. Da normalerweise die $v$ NH höher liegt, z. B. $\left.\left.\mathrm{H}_{2} \mathrm{~B}-\mathrm{NH}-\mathrm{NH}-\mathrm{BH}_{2} 3195^{9}\right), \mathrm{CH}_{3}-\mathrm{NH}-\mathrm{NH}-\mathrm{CH}_{3} 3223,3294^{10}\right)$ und $\left(\mathrm{CH}_{3}\right)_{2} \mathrm{NH}$ $3355 \mathrm{~cm}^{-111}$ ), so wurde die höchste beobachtete Frequenz der $v$ NH zugeordnet. Weiterhin gehört die jeweils niedrigste Linie im Bereich $800-900 \mathrm{~cm}^{-1}$ zur Valenzschwingung $v$ NN.

в) K. W. F. Konlrausch, Raman-Spektren, Akad. Verlagsges. Leipzig 1943.

${ }^{7}$ ) G. Radinger u. H. Wittek, Z. physik. Chem. Abt. B 45, 329 (1940).

8) Diplomarbeit G. Herrmann, Technische Hochschule Stuttgart 1961.

${ }^{9}$ ) J. Goubrats u. E. Rickkr, Z. anorg. allg. Chem. 810, 123 (1961).

10) D. W. E. Axford, G. J. JaNZ u. K. E. Russell, J. chem. Physies 19, 704 (1951).

11) J. R. Barcelo u. J. Bellanato, Spectrochim. Acta 8, 27 (1956/57). 
Tabelle 2

Die Schwingungsspektren von Hydrazin, Natrium- und Zinkhydrazid und ihre Zuordnung

\begin{tabular}{|c|c|c|c|c|c|c|}
\hline & $\begin{array}{c}\mathrm{N}_{2} \mathrm{H}_{4} \\
\text { gasförmig }\end{array}$ & $\begin{array}{l}\mathrm{N}_{2} \mathrm{H}_{4} \\
\text { flüssig }\end{array}$ & $\begin{array}{l}\mathrm{N}_{2} \mathrm{H}_{4} \\
\text { fest }\end{array}$ & $\mathrm{Na}+\mathrm{N}_{2} \mathrm{H}_{8}^{-}$ & $\mathrm{Zn}\left(\mathrm{N}_{2} \mathrm{H}_{3}\right)_{2}$ & Zuordnung \\
\hline & \multirow{4}{*}{ 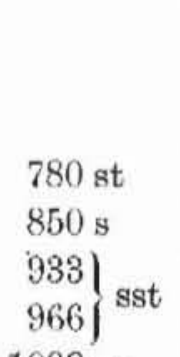 } & \multirow{3}{*}{$\begin{array}{l}800 \\
873\end{array}$} & \multirow[b]{2}{*}{795} & \multirow{4}{*}{$\begin{array}{l}847 \mathrm{~m} \\
972 \mathrm{~s} \\
988 \mathrm{~s}\end{array}$} & $\left.\begin{array}{l}409 \text { st } \\
473 \text { st } \\
593 \mathrm{~m}\end{array}\right\}$ & $\begin{array}{l}v \mathrm{Zn}-\mathrm{N} \\
\delta \mathrm{ZnNHod} . \tau\end{array}$ \\
\hline$\delta_{8} \mathrm{NXH}_{2}$ & & & & & & \\
\hline$v \mathrm{NN}$ & & & $\begin{array}{r}885 \\
1072\end{array}$ & & 908 st & $\begin{array}{l}v \text { NN } \\
\delta \text { NNH }\end{array}$ \\
\hline$\delta \mathrm{NNH}_{2}$ & & 1038 & 1078 & & $960 \mathrm{~s} \mathrm{Sch}$ & $0 \mathrm{NND}_{2}$ \\
\hline$\gamma \mathrm{NH}_{2}$ & $1098 \mathrm{~m}$ & & 1124 & $\begin{array}{l}1103 \text { sst } \\
1132 \text { Sch }\end{array}$ & $\begin{array}{l}1069 \mathrm{st} \\
.11 .57 \mathrm{~m}\end{array}$ & $\delta$ NNH \\
\hline & $1275 \mathrm{~s}$ & 1280 & 1320 & $\begin{array}{l}1232 \mathrm{~s} \\
1330 \mathrm{~s} \text { Sch }\end{array}$ & $\begin{array}{l}1271 \mathrm{~m} \text { Sch } \\
1362 \mathrm{~m}\end{array}$ & $\gamma \mathrm{NH}_{2}$ \\
\hline$\delta_{\mathrm{B}} \mathrm{NH}_{2}$ & 1493 ss & & & 1590 ot & 1582 ot & \\
\hline$\delta_{\mathrm{as}} \mathrm{NH}_{2}$ & & & & 1599 st & $1583 \mathrm{st}$ & $\delta \mathrm{NH}_{2}$ \\
\hline$v \mathrm{NH}_{2}$ & $\left.\begin{array}{l}3280 \\
3314 \\
3325\end{array}\right\} \mathrm{m}$ & $\begin{array}{l}3182 \\
3241 \\
3265\end{array}$ & 3240 & $\begin{array}{l}3100 \mathrm{~m} \\
3155 \mathrm{~m} \\
3202 \mathrm{~s}\end{array}$ & $\begin{array}{l}3085 \mathrm{~m} \\
3190 \mathrm{st} \mathrm{m}\end{array}$ & $\begin{array}{l}v_{8} \mathrm{NH}_{2} \\
v_{\text {as }} \mathrm{NH}_{2}\end{array}$ \\
\hline & 3350 J & 3328 & 3315 & & & \\
\hline
\end{tabular}

Etwas schwieriger ist die Zuordnung der Deformationsschwingungen. Von den fünf möglichen ist normalerweise die Torsionsschwingung schwer beobachtbar, so daß vier zu erwarten sind. Aus den Spektren von Hydrazin ${ }^{12}$ ), Hydroxylamin $\left.{ }^{13}\right), \mathrm{CH}_{3} \mathrm{~N}_{2} \mathrm{H}_{3}{ }^{10}$ ) sind die Schwingungen der $\mathrm{NH}_{2}$-Gruppe bei folgenden Frequenzen zu erwarten: $\delta \mathrm{NH}_{2} 1500-1600$, $\delta \mathrm{NNH}_{2}, 1100-1200$ und $\delta \mathrm{NNH} 800-1000 \mathrm{~cm}^{-1}$. Entsprechend kann aus den Spektren von $\mathrm{CH}_{3} \mathrm{~N}_{2} \mathrm{H}_{3}{ }^{10}$ ), Pyrrol ${ }^{14}$ ) und $\mathrm{HN}_{3}{ }^{15}$ ) auf einen Wert von $\delta \mathrm{NNH} 1100-1150 \mathrm{~cm}^{-1}$ geschlossen werden. Da aus der Lage der NH-Valenzschwingungen geschlossen werden darf, da $B$ beim Utbergang von den Vergleichssubstanzen zu den Hydraziden keine wesentlichen Änderungen in den NH-Bindungsverhältnissen auftreten, so ergibt sich die in Tab. 2 getroffene Zuordnung. Dabei zeigt sich jedoch, daß die $\mathrm{NH}_{\mathbf{2}}$-Deformationsschwingungen teilweise aufspalten und zwar ziemlich stark. Eine derartige Aufspaltung der Deformationsschwingungen wird häufiger beobachtet. Sie kann auf Gittereffekte, z. B. mehrere Formeleinheiten in der Elementarzelle, zurückgeführt werden, oder auf Assoziationen. Die Größe der Aufspaltung $\delta \mathrm{NNH}_{2} 16, \gamma \mathrm{NH}_{2} 98$ spricht eher für die zweite Erklärung. Sieht man von Frequenzverschiebungen ab, so ergibt sich für die Zinkverbindung ein

12) P. A. Gigìure u. J. D. LiU, J. chem. Physics 20, 136 (1952); W. Fresenrưs u. J. Karweil, Z. physik. Chem. Abt. B 44, 1 (1939); J. Goubeau, Z. physik. Chem. Abt. B 45, 237 (1940).

13) R. E. Nightingale u. E. L. Wagner, J. chem. Physics 22, 203 (1954).

14) R. C. Lord jun. u. F. A. Mrller, J. chem. Physies 10, 328 (1942).

15) E. H. Eyster u. R. H. Grllette, J. chem. Physics 8, 369 (1940). 
sehr ähnliches Spektrum mit derselben Zuordnung. Zusätzlich werden im $\mathrm{CsBr}$-Bereich noch einige Linien aufgefunden, von denen 409 und $473 \mathrm{~cm}^{-1}$ zu Schwingungen von $\mathrm{ZnN}$ Komplexen gehören, da auch in $\mathrm{Zn}\left(\mathrm{NH}_{3}\right)_{2} \mathrm{Cl}_{2}$ und $\left.\mathrm{Zn}\left(\mathrm{NH}_{3}\right)_{4} \mathrm{~J}_{2}{ }^{16}\right)$ Schwingungen bei 422 bzw. $410 \mathrm{~cm}^{-1}$ auftreten. Die noch verbleibende Bande bei $593 \mathrm{~cm}^{-1}$ ist entweder eine Torsionsschwingung, da diese im vergleichbaren festen Hydroxylamin bei $535 \mathrm{~cm}^{-1}$ liegt, oder es handelt sich um eine Deformationsschwingung $\delta \mathrm{ZnNH}$, die in dieser Gegend zu erwarten ist.

\section{Folgerungen}

Fin klares Bild ergeben die Kraftkonstanten $\mathrm{k}_{\mathrm{NN}}$, die aus den $v_{\mathrm{N} .}$ der einzelnen Hydrazinverbindungen nach dem einfachen Ansatz einer zweiatomigen Molekel sich ergeben (Tab. 3).

Es resultiert zwar für das Hydrazidion im Natriumsalz der niedrigste Wert. Er unterscheidet sich jedoch nur wenig vom Wert für das gasförmige Hydrazin. Nun konnte schon früher ${ }^{12}$ ) beim Hydrazin eine sehr starke Beeinflus-

Tabelle 3

Kraftkonstanten verschiedener Hydrazinverbindungen

\begin{tabular}{l|c|c|c|c}
\hline & $\begin{array}{c}\text { Aggregat- } \\
\text { zustand }\end{array}$ & $\begin{array}{c}\text { freie } \\
\text { Elektronen- } \\
\text { paare }\end{array}$ & $\begin{array}{c}\text { Frequenz } \\
\mathrm{v}_{\mathrm{NN}} \mathrm{cm}^{-1}\end{array}$ & $\mathrm{k}_{\mathrm{NN}} \frac{\mathrm{mdyn}}{\AA}$ \\
\hline $\mathrm{N}_{2} \mathrm{H}_{3}^{-}$ & fest & 3 & 847 & 3,35 \\
$\mathrm{~N}_{2} \mathrm{H}_{\mathbf{4}}$ & gasförmig & 2 & 850 & 3,48 \\
& flüssig & 2 & 873 & 3,68 \\
& fest & 2 & 885 & 3,77 \\
$\mathrm{~N}_{2} \mathrm{H}_{5}^{+}$ & fest & 1 & 973 & 4,71 \\
$\mathrm{~N}_{2} \mathrm{H}_{\mathbf{6}}{ }^{2+}$ & fest & 0 & 1024 & 5,37
\end{tabular}
sung des Spektrums durch zwischenmolekulare Kräfte - vor allem Wasserstoffbrücken - nachgewiesen werden, die sich auch in den verschiedenen Aggregatzuständen deutlich auswirken (Tab. 2). Mit zunehmender Wasserstoffbrückenbindung, kenntlich an der Abnahme der NH-Valenzfrequenzen, nimmt $k_{\mathrm{VN}}$ zu. Die freien Elektronenpaare ändern dabei ihren Charakter im Sinne einer zunehmenden Bindungsbeanspruchung. Da in beiden Hydraziden die $v$ NH sehr tief liegen, teilweise unter den Werten im festen Hydrazin, so muß, besonders im Natriumhydrazid, auf starke Wasserstoffbrücken geschlossen werden, so daß die Kraftkonstante von festem Hydrazin als Vergleich herangezogen werden muß. Damit ergibt sich dann eine Verringerung vom Hydrazin zum Hydrazid von rund $10 \%$, die der üblichen Abnahme bei der Zunahme von freien Elektronenpaaren entspricht, die sich aus den Beispielen der Tab. 4 durchschnittlich zu 15\% ergibt.

In bezug auf die Kraftkonstante $\mathrm{k}_{\mathrm{NN}}$ unterscheidet sich das Zinkhydrazid sehr stark vom Natriumsalz. Nimmt man ebenfalls das Ion $\mathrm{N}_{2} \mathrm{H}_{3}^{-}$an, so resultiert $\mathrm{k}_{\mathrm{NN}}=3,85 \mathrm{mdyn} / \AA$, ein Wert, der höher liegt als

16) G. M. Barrow, R. H. Kruterer u. F. Basolo, J. inorg. nucl. Chem. 2, 340 (1956). 
Tabelle 4

Kraftkonstanten in mdyn/ $\Lambda$ von Molekeln und Ionen mit versehiedener ZahI von freien Elektronenparen

\begin{tabular}{|c|c|c|c|c|c|c|}
\hline $\begin{array}{c}\text { freie } \\
\text { Elektronen- } \\
\text { paare }\end{array}$ & $\left.\mathrm{k} \mathrm{CC}^{17}\right)$ & $\mathrm{k} N \mathrm{~N}$ & & k NO & $\mathrm{k} N \mathrm{O}$ & k 00 \\
\hline $\begin{array}{l}0 \\
1 \\
2 \\
2,5 \\
3 \\
3,5 \\
4 \\
5\end{array}$ & $\begin{array}{ll}\mathrm{C}_{2} \mathrm{H}_{2} & 15,7 \\
\mathrm{C}_{2} \mathrm{H}^{-} & 13,1\end{array}$ & $\begin{array}{l}\mathrm{N}_{2} \mathrm{H}_{3}^{2+} \\
\mathrm{N}_{2} \mathrm{H}_{3}^{+} \\
\mathrm{N}_{2} \mathrm{H}_{4 \text { fest }} \\
\mathrm{N}_{2} \mathrm{H}_{8}^{-}\end{array}$ & $\begin{array}{l}5,4 \\
4,7 \\
3,8 \\
\\
3,4\end{array}$ & $\begin{array}{l}\mathrm{NH}_{3} \mathrm{OH}+4,9 \\
\mathrm{NH}_{2} \mathrm{OH} \quad 4,0\end{array}$ & $\begin{array}{ll}\mathrm{RNO}_{2} & 9,7 \\
\mathrm{NO}_{2}^{-} & 8,0\end{array}$ & $\begin{array}{ll}\mathrm{H}_{2} \mathrm{O}_{2} & 4,0 \\
\mathrm{HO}_{2}^{-} & 3,7\end{array}$ \\
\hline
\end{tabular}

alle Werte des Hydrazins. Dieser Widerspruch gegenüber den Erwartungen ist jedoch einfach zu erklären. Die im Zinkhydrazid beobachteten Frequenzen im Gebiet $400-500 \mathrm{~cm}^{-1}$ sprechen eindeutig für eine kovalente Bindung $\mathrm{Zn}-\mathrm{N}$, d. h. ein freies Elektronenpaar des Hydrazid-Ions verschwindet, so daß sicher die Elektronenkonfiguration des Hydrazins mit nur zwei freien Elektronenpaaren vorliegt. Da durch diese Bindung am Zink die schwingende Masse des Stickstoffs erhöht wird, kann nicht mehr mit dem einfachen Zweimassenmodell gerechnet werden. Daraus folgt mit Sicherheit, daß die Kraftkonstanten über $3,85 \mathrm{mdyn} / \AA$ liegt, d. h. höher wie im. Hydrazin ist und sich dem Hydrazonium-Ion nähert. Dies genügt zu der weiteren Annahme, daß3 das freie Elektronenpaar der $\mathrm{NH}_{2}$-Gruppe koordinativ an das Zink gebunden ist, so daß folgende Verknüpfungen angenommen werden können:

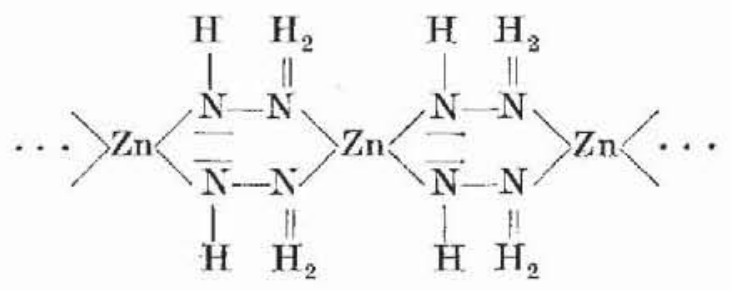

Dies ist nur eine der zahlreichen Möglichkeiten der Verknüpfung, die auch zu Raumnetzstrukturen führen können. Mit großer Wahrscheinlichkeit läßt sich jedoch der Schluß ziehen, daß das Zink von vier Stickstoffatomen tetraedrisch umgeben ist. Weiterhin beweist das erhaltene Debyeogramm, daß 3 eine regelmäßige Anordnung resultiert.

Dieser Unterschied in den Bindungsverhältnissen äußert sich auch deutlich im Reaktionsvermögen der beiden Hydrazide. Das Natrium-

17) J. Goubenu u. O. Betrer, Z. anorg. allg. Chem. 310, 110 (1961). 
hydrazid ist eine äußerst instabile Substanz. Während sie sich im reinsten Stickstoff als nicht stoß- und schlagempfindlich erwies, trat beim Reiben mit Nujol im Achatmörser in Gegenwart von geringen Mengen Sauerstoff Explosion ein. Aufnahmen in Hostaflon konnten nicht ausgeführt werden, da Gemische von Natriumhydrazid mit Hostaflon zu heftigen Explosionen unter vollständiger Zertrümmerung der Gefäße Anlaß gaben. ÄhnlicheReaktionen wurden bei Zinkhydrazid nicht beobachtet. Es zeigt sehr viel größere Stabilität wegen der ganz andersartigen Bindung des Hydrazins. Die Kraftkonstante der $\mathrm{N}-\mathrm{N}$-Bindung ist deshalb ein guter Maßstab für die Stabilität der Hydrazinverbindungen.

\section{Experimenteller Teil $^{1}$ ) \\ Darstellung von Natriumhydrazid}

Die Darstellung erfolgte im großen und ganzen nach der Vorschrift von ScHLENk und WEICHSELFELDER ${ }^{3}$ ). Es wurde immer in Stickstoffatmosphäre gearbeitet. Das als ,wasserfrei" bezogene Hydrazin $(30 \mathrm{~g})$ wurde mit Natrium unter einer Benzolschicht $(1 \mathrm{~cm})$ vollständig entwässert, kenntlich an der Beständigkeit der blaßgelben Farbe von gelöstem Natriumhydrazid. Unter Luft- und Feuchtigkeitsausschluß wurden Benzol und Hydrazin in den Reaktionskolben unter vermindertem Druck destilliert. Darin wurden im strömenden Stickstoff $0,8 \mathrm{~g}$ blankes Natrium in fünf Teilen im Laufe von mehreren Stunden unter ständigem Schütteln eingetragen. Die zunächst am Metall auftretende blaue Farbe verschwindet mit dem Auflösen des Metalls. Nach Beendigung der Umsetzung wird aus der klaren, nun tiefgelb gefärbten Lösung das Benzol und das überschüssige Hydrazin im Vakuum abdestilliert, wobei die Temperatur zum Schlu $\beta$ für ${ }^{1} / 2$ Stunde auf $60^{\circ}$ gesteigert wurde. Das Hydrazid fiel als glänzende, weiße, in dicker Schicht blaßgelbe, Blättchen an.

Das Salz war unter Stickstoff aufbewahrt über längere Zeit stabil. Zur Analyse wurden kleinere Mengen mit Benzol überschichtet und zunächst mit Methylalkohol und schließlich mit Wasser zu Hydrazin und $\mathrm{NaOH}$ hydrolysiert. Das Benzol wurde im Scheidetrichter abgetrennt. Die wäßrige, methylalkoholische Schicht mit verdünnter Schwefelsäure angesäuert und der Methylalkohol weggekosht. In dieser Lösung wurde das Hydrazin jodometrisch nach SToLc⿱亠𧘇 ${ }^{18}$ ) mit Stärke als Indikator und das Natrium als Natriumsulfat bestimmt.

$$
\begin{array}{lll}
\mathrm{Na}_{2} \mathrm{~N}_{2} \mathrm{H}_{3}: & \mathrm{Na} & \text { ber.: } 42,6 \% \text {; gef.: } 41,9 ; 42,5 \% \text {; } \\
& \mathrm{N}_{2} \mathrm{H}_{3}^{-} & \text {ber.: } 57,4 \% \text {; gef.: } 49,4 ; 49,1 ; 49,5 \% .
\end{array}
$$

Der niedrige Hydrazinwert erklärt sich zwanglos durch die nicht vollständige Extraktion des $\mathrm{N}_{2} \mathrm{H}_{4}$ aus dem Benzol. Da das IR-Spektrum keinen Anhaltspunkt für Verunreinigungen, z. B. $\mathrm{NaOH}$ ergibt und da der Natriumwert dem theoretischen entspricht, so besteht kein Zweifel, daß ein reines Mydrazid vorliegt.

\section{Darstellung des Zinkhydrazids}

Diese erfolgte nach den Angaben von EBLER und KRAUSE ${ }^{4}$ ) durch Umsetzung von Zinkdiäthyl mit wasserfreiem Hydrazin. Das erstere wurde nach Noller und Lachman. ${ }^{19}$ )

18) R. SToLlÉ, J. prakt. Chem. (2) 66, 334 (1902).

19) C. R. Nollier u. Mitarb., J. Amer. chem. Soc. 51, 594 (1929). 
aus Äthyljodid und Zink-Kupferlegierung erhalten. Dieses wurde nach der Auswarge (4 g) mit Äther verdünnt. Zu dem bei dem Natriumhydrazid abdestilliertem wasserfreien Hydrazin $(1,95$ g) wurde Äther zugegeben und diese Lösung im Laufe einer halben Stunde zu Zinkdiäthyllösung zugetropft, alle Reaktionen unter Stickstoffatmosphäre. Gleichzeitig mit der Gasentwicklung bildete sich langsam ein weißer, teilweise blaßgelblicher Niederschlag. Nach dem Zutropfen setzte sich die Niederschlagsbildung unter Gasentwicklung fort und war ungefähr nach einer Stunde beendet. Das Produkt wurde unter Stickstoff abfiltriert und dreimal mit Äther ausgewaschen und schließlich der Äther und die letzte Spur Hydrazin im Stickstoffstrom und im Vakuum entfernt.

Das Zinkhydrazid zeigte im ätherfeuchten Zustand blaßgelbe Farbe, trocken war es fast rein weiß. Die Vorbereitung zur Analyse erfolgt in der beim Natriumhydrazid beschriebenen Art und Weise, ebenso die Bestimmung des Hydrazins. Die Zinkbestimmung erfolgte komplexometrisch nach SCHWARZEABACH ${ }^{20}$ ) und teilweise gravimetrisch als Zinksulfat: nach $\left.\operatorname{EuLIR}^{21}\right)$.

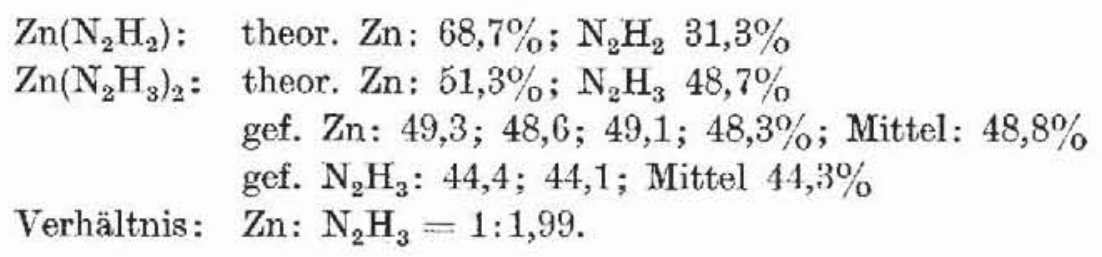

Der Fehlbetrag der Analyse ist auf einen geringeren Gehalt von Zinkjodid zurückzuführen.

Das so erhaltene Zinkhydrazid reagiert nicht, wie das von EbLER und Krause beschriebene, an feuchter Luft mit Feuererscheinung, sondern es klumpt innerhalb weniger Sekunden zusarnmen und beginnt zu zerfließen, wobei Hydrazingeruch auftritt, d. h. es tritt in der Hauptsache Hydrolyse ein. Mit Wasser bildet sich dementsprechend Hydrazin und schwerlösliches Zinkhydroxid. Als hochmolekulare Substanz ist es in Benzol, Äther, $\mathrm{CCl}_{4}$ unlöslich; auch mit Methylalkohol tritt innerhalb einer Stunde keine merkliche Reaktion ein. In Hydrazin ist es löslich, wahrscheinlich wird das Hydrazid-Ion partiell aus der Komplexsphäre durch das Hydrazin verdrängt und tritt nur noch als einzähliger Ligand auf. Das mit $\mathrm{CuK}_{\alpha}$-Strahlung bei einer Belichtungszeit von $21 / 2$ Stunden erhaltene, schwache Debyeogramm ist in Tab. 5 wiedergegeben:

Tabelle 5

Debyeogramm von $\mathrm{Zn}\left(\mathrm{N}_{2} \mathrm{H}_{3}\right)_{2}$

\begin{tabular}{c|c|c|c|c|c|c|c|c|c|c}
\hline $\mathrm{Nr}$. & 1 & 2 & 3 & 4 & 5 & 6 & 7 & 8 & 9 & 10 \\
\hline $2 \vartheta$ & 9,5 & 13,5 & $\begin{array}{c}14,5 \\
\mathrm{~s}\end{array}$ & $\begin{array}{c}16,0 \\
\mathrm{st}\end{array}$ & $\begin{array}{c}18,5 \\
\mathrm{~m}\end{array}$ & $\begin{array}{c}21,0 \\
\mathrm{ss}\end{array}$ & $\begin{array}{c}22,5 \\
\mathrm{ss}\end{array}$ & $\begin{array}{c}28,5 \\
\mathrm{ss}\end{array}$ & $\begin{array}{c}31,5 \\
\mathrm{~m}\end{array}$ & $\begin{array}{c}34,5 \\
\mathrm{~m}\end{array}$ \\
Intensität & $\mathrm{s}$ & $\mathrm{m}$ & $\mathrm{s}$
\end{tabular}

Einer hochsymmetrischen Kristallklasse konnte es nicht zugeordnet werden.

\section{Versuche zur Darstellung von Caleiumhydrazid}

In Übereinstimmung mit früheren Versuchen von WELSH ${ }^{2}$ ) und FEH ḱR ${ }^{22}$ ) wurde festgestellt, daß Hydrazin mit metallischem CaIcium bis $75^{\circ}$ nicht reagiert. Dagegen war zu

20) G. Sohwarzenbach, Die komplexometrische Titration. 2. Aufl. Stuttgart 1956.

21) W. Eutar, Z. anorg. Chem. 25, 146 (1900).

22) F. Fent́r, J. Cremer u. W. Fromm, Z. anorg. allg. Chem. 287, 175 (1956). 
erwarten, daß Caleiumamid oder Calciumhydrid mit Hydrazin reagieren. Die letztere Reaktion wurde untersucht. In Äther aufgeschlämmtes Calciumhydrid wurde mit wasserfreiem Hydrazin versetzt. Dabei tritt meistens sofort eine lebhafte Gasentwicklung ein, die erst nach vollständigem Verbrauch des Hydrids aufhört. Gleichzeitig entsteht in der Hydrazinphase ein weißer, voluminöser Niederschlag, der noch genauer untersucht werden soll. Da mit Überschuß von Hydrazin gearbeitet wurde, dürfte es sich um $\mathrm{Ca}\left(\mathrm{N}_{2} \mathrm{H}_{3}\right)_{2}$ handeln.

\section{Infrarot-Spektren}

Die Aufnahmen erfolgten mit einem Phrkin-Elmar-Spektrophotometer, Modell 21 im NaCl- und CsBr-Bereich. Da beim Zusammenreiben von Natriumhydrazid mit Nujol in einer Achatreibschale im Stickstoffkasten Explosionen erfolgten, so wurden die Mischungen in einem besonderen Gefäß unter dauerndem Durchströmen mit reinstem Stickstoff dargestellt. Diese Pasten konnten dann ohne Zersetzungserscheinungen im Stickstoffkasten zwischen die Kochsalzscheiben gebracht werden und die Aufnahmen in einer luftdicht schließenden Zelle ohne Störung durchgeführt werden. Da Mischungen von Natriumhydrazid mit Hostaflon zu heftigen Explosionen führten, so wurden Nujolaufnahmen mit Kompensation des Nujols durchgeführt. Das in Tab. 2 angegebene Spektrum von Natriumhydrazid wurde aus vier Aufnahmen, davon zwei mit Kompensation, gemittelt, die Werte für Zinkhydrazid sind im $\mathrm{NaCl}$-Bereich Mittelwerte aus sechs, im $\mathrm{CsBr}$-Bereich aus vier Aufnahmen.

Wir danken der Deutschen Forschungsgemeinschaft und dem Fonds der Chemie für die Unterstützung dieser Arbeit.

Stuttgart, Laboratorium für anorganische Chemie der Technischen Hochschule.

Bei der Redaktion eingegangen am 1. Februar 1962. 